\title{
Semi-implicit semi-Lagrangian modelling of the atmosphere: a Met Office perspective*
}

\author{
Tommaso Benacchio ${ }^{1 *}$, Nigel Wood $^{1}$ \\ ${ }^{1}$ Met Office, Exeter, UK \\ *Email address for correspondence: tommaso.benacchio@metoffice.gov.uk \\ Communicated by Luca Bonaventura \\ Received on June 15, 2015. Accepted on August 2, 2015.
}

\begin{abstract}
The semi-Lagrangian numerical method, in conjunction with semi-implicit time integration, provides numerical weather prediction models with numerical stability for large time steps, accurate modes of interest, and good representation of hydrostatic and geostrophic balance. Drawing on the legacy of dynamical cores at the Met Office, the use of the semi-implicit semi-Lagrangian method in an operational numerical weather prediction context is surveyed, together with details of the solution approach and associated issues and challenges. The numerical properties and performance of the current operational version of the Met Office's numerical model are then investigated in a simplified setting along with the impact of different modelling choices.

Keywords: Semi-Lagrangian method, semi-implicit method, dynamical core, normal mode analysis

AMS subject classification: 65N06, 65N12, 65N22

\section{Introduction}

Many - perhaps most - of the operational numerical weather prediction (NWP) models employ a combination of semi-implicit (SI) and semi-Lagrangian (SL) discretizations (that combination is referred to as SISL). Examples of such models are IFS [1], in use at the European Centre for Medium-Range Weather Forecasts (ECMWF); ARPEGE [2], at Météo-France; GEM [3], at the Canadian Meteorological Centre (CMC); GRAPES [4], at China Meteorological Administration (CMA); the Japan Meteorological Agency (JMA, [5])'s model; SL-AV [6], at Russia's Roshydromet; GFDL's HIRAM [7] and NCAR's CAM-FV [8] in the US, as well as the Met Office's Unified Model [9] in use, in addition to the UK, at the Australian Bureau of Meteorology and at the Korea Meteorological Administration.
\end{abstract}

*CBritish Crown Copyright Met Office 2016 


\section{SISL modelling: a Met Office perspective}

The semi-Lagrangian scheme is essentially a Lagrangian advection scheme applied over each time step in which the advection of a quantity is evaluated by tracing back from a mesh point to where an assumed parcel of air originated at the previous time step (the departure point). The advected quantity is then evaluated by interpolation to that departure point using values of the quantity at surrounding mesh points at the previous time step. It is termed semi-Lagrangian because it is only Lagrangian for the duration of the time step - the parcel of air is constrained to always arrive at a mesh point at the end of the time step. The authors of [10] give a comprehensive review of the history, use and benefits of the semi-Lagrangian scheme.

The semi-implicit scheme is a time integration method that averages tendencies over one or more time steps, with the average involving unknown quantities at the next time level. In its two-time-level, time-centred form, it is equivalent to the Crank-Nicholson scheme. It is termed semi-implicit because the time-averaging is traditionally applied only to a specific selection of terms. For example, non-linear terms are traditionally separated into a linear component that is evaluated implicitly and a non-linear residual that is evaluated explicitly. As will be discussed in this article more recent incarnations of the scheme apply the time averaging more consistently to all the terms and solve the resulting non-linear equations iteratively. For these schemes, perhaps iterative-implicit is a more suitable term, e.g. [11].

It is common for large scale geophysical models to combine the SI scheme with the SL scheme because in the resulting SISL scheme advection by the meteorologically important jets is stably and accurately handled by the semi-Lagrangian method whilst the modes of oscillation (Rossby waves, gravity waves, acoustic waves, and their variants) are stably handled by the semi-implicit method. For the SI scheme the accuracy of each mode of oscillation is determined by how well it is resolved by the chosen time step. This is particularly important for non-hydrostatic compressible models that admit acoustic modes, which are known by themselves not to be of meteorological importance (although the effects of compressibility on other modes might be) yet have extremely small time scales. In contrast Rossby modes are very important to the accuracy of a forecast but have significantly longer time scales. It is therefore of considerable benefit to employ a scheme that allows the time step to be chosen to resolve the important modes whilst being stable (but not accurate) for the modes that are not resolved.

There are many benefits that accrue from the SISL combination of these schemes and their excellent stability properties. First, time steps can be used that are large enough so that the temporal discretization errors are of the same order as the spatial discretization errors [10]. Second, numerical stability permits the efficient use of global latitude-longitude meshes 


\section{T. Benacchio, N. Wood}

(with their clustering of mesh points near to the poles). This itself brings advantages in terms of accuracy and efficiency. We refer to [12] for a comprehensive review of the pros and cons of various choices for global meshes. A further, and perhaps less well known, benefit is the natural way in which an SI scheme can preserve the underlying balanced components of large scale geophysical flows, such as geostrophic and hydrostatic balance [13].

The Met Office started experimenting with semi-Lagrangian schemes in the early 1990s [14]. This work built on experience with a semi-implicit scheme used for the Met Office's mesoscale model [15] and led to the development of the SISL dynamical core known as the New Dynamics [16]. (Here "dynamical core" is taken to mean that part of the numerical model that governs the evolution of resolved atmospheric fluid dynamical processes.) The New Dynamics became the operational dynamical core of the Met Office's Unified Model (UM) in 2002. By that time the SISL approach had been reasonably widely applied to the hydrostatic primitive equations (HPEs). However, the development of the New Dynamics faced a number of new challenges:

- Following the work of [17] the UM does not make the shallowatmosphere approximation and retains the full Coriolis terms. This choice is in contrast to the HPEs which make both the shallowatmosphere approximation and the traditional approximation to the Coriolis terms. We refer to [18] for a discussion of approximate equation sets.

- The Unified Model is so termed because the same dynamical core is used for all model configurations, from high resolution numerical weather prediction to long term climate simulations [9], including the mesoscale model configuration for which non-hydrostatic effects are important. Application of the SISL scheme to a nonhydrostatic model was a significant departure from its usual application to the HPEs, and was contemporary with similar development in the CMC's model GEM [3].

- Associated with not making the hydrostatic approximation the model uses a height-based vertical coordinate in contrast to the usual pressure-based ones. Although [19] had proposed a pressurebased coordinate for use in a non-hydrostatic model, this was not appropriate for a deep-atmosphere model, i.e. one that does not make the shallow approximation. Since then the authors of [20] generalized Laprise's approach by recognizing that in a shallow-atmosphere model pressure and mass are interchangeable, whereas this is not the case in a deep-atmosphere model, see [21]. If height is not used 
in a deep-atmosphere model, then mass, as opposed to pressure, is the appropriate variable to use as a vertical coordinate.

- The long term climate simulations required excellent mass conservation which at the time of development of New Dynamics (i.e. the late 1990's [14]) was a challenge. We refer to [22] for in-depth discussion on mass conservation issues.

Overcoming these challenges was a considerable achievement by the designers of the New Dynamics [14] but, by necessity of what was understood at the time, the design had a number of shortcomings. Addressing these issues led to the development of ENDGame (Even Newer Dynamics for General atmospheric modelling of the environment, [23]), which became the operational dynamical core of the UM in 2014. Over the 20 years since the New Dynamics, ENDGame has exploited the key development on efficient iterative approaches to the solution of the SISL equations. The use of these approaches was pioneered at Environment Canada and is summarized in a nonhydrostatic setting in [24]. The technique was explored in the ECMWF model by [25] and in a New Dynamics setting by [26].

By considering the example of ENDGame, this paper aims to describe some of the characteristics of a SISL method in the context of operational NWP. Section 2 describes the general SISL approach, before focusing on the iterative approach and the departure point calculation. The features of the numerical scheme are then demonstrated in a simplified vertical column setting in section 3, where related results with particular modelling choices are also presented. Section 4 contains some concluding remarks.

\section{The semi-implicit semi-Lagrangian approach}

\subsection{The general approach}

Consider the equation

$$
\frac{D F}{D t}=G,
$$

where $F=F(\boldsymbol{x}, t)$, and $G=G(\boldsymbol{x}, t)$ are generic scalar or vector variables, and $\frac{D}{D t}(\cdot)=\frac{\partial}{\partial t}(\cdot)+\mathbf{u} \cdot \nabla(\cdot)$ denotes the material derivative. The semiimplicit discretization of (1) is obtained by first integrating it in time along a trajectory $\boldsymbol{x}=\boldsymbol{x}(t)$ to obtain:

$$
F[\boldsymbol{x}(t+\Delta t), t+\Delta t]-F[\boldsymbol{x}(t), t]=\int_{t}^{t+\Delta t} G\left[\boldsymbol{x}\left(t^{\prime}\right), t^{\prime}\right] d t^{\prime} .
$$




\section{T. Benacchio, N. Wood}

Then, in a centred, second-order accurate scheme the right-hand side is approximated using the trapezoidal rule, giving:

(3)

$F[\boldsymbol{x}(t+\Delta t), t+\Delta t]-F[\boldsymbol{x}(t), t]=\frac{\Delta t}{2}\{G[\boldsymbol{x}(t+\Delta t), t+\Delta t]+G[\boldsymbol{x}(t), t]\}$.

In a semi-Lagrangian scheme $\boldsymbol{x}(t+\Delta t)$ is denoted as $\boldsymbol{x}_{A}$, the arrival point which is constrained to be a mesh point, and $\boldsymbol{x}(t)$ is denoted as $\boldsymbol{x}_{D}$, the departure point which will in general not be a mesh point. Additionally using superscript $n$ to denote evaluation at time $t$ and $n+1$ to denote time $t+\Delta t$, expression (3) can be rearranged as

$$
\left(F-\frac{\Delta t}{2} G\right)_{A}^{n+1}=\left(F+\frac{\Delta t}{2} G\right)_{D}^{n} .
$$

When applied to a full model, $F$ represents the model state vector, and in general $G$ will be a non-linear function of $F$. The challenge is then how to solve (4) for $F^{n+1}$. This is usually achieved by linearizing $G$ in some manner, either at the spatially continuous or spatially discrete level.

\subsection{Some specific issues}

As a specific example of how $G$ might be linearized, let $G$ represent the vertical component of the pressure gradient term which, using the ENDGame choice of prognostic variables, is

$$
G_{A}^{n+1} \equiv c_{p} \theta_{A}^{n+1} \delta_{z} \Pi_{A}^{n+1} .
$$

Above, $c_{p}$ denotes the specific heat at constant pressure, $\Pi=\left(p / p_{0}\right)^{R / c_{p}}$ is the Exner function, $R$ the gas constant, and $\theta$ the potential temperature, i.e., the temperature a fluid parcel attains if transported isentropically at sea-level pressure $p_{0}$ to a height with pressure $p$. In (5) $\delta_{z}$ denotes either the continuous or discrete vertical derivative.

If the traditional semi-implicit approach (i.e. that which is usually applied to the HPEs) is followed then a semi-implicit reference profile is chosen that is fixed in time for both $\theta$ and $\Pi$, i.e. $\theta_{r e f}$, and $\Pi_{r e f}$. Note that in the HPE context it is usual to choose the reference profile to be isothermal (and also to write the pressure gradient term in terms of temperature $T$ and $\operatorname{logarithm}$ of pressure $\log p$ ). If the perturbations from the reference profiles are denoted by a prime superscript then expression (5) is rewritten exactly as:

$$
G=c_{p} \theta_{r e f} \delta_{z} \Pi_{r e f}+c_{p} \theta_{r e f} \delta_{z} \Pi^{\prime}+c_{p} \theta^{\prime} \delta_{z} \Pi_{r e f}+c_{p} \theta^{\prime} \delta_{z} \Pi^{\prime}
$$




\section{SISL modelling: a Met Office perspective}

A tractable linear problem in the unknowns $\theta^{n+1}$ and $\Pi^{n+1}$ can then be obtained by evaluating the two linear terms using time level $n+1$ for the primed quantities but evaluating the non-linear residual term, $c_{p} \theta^{\prime} \delta_{z} \Pi^{\prime}$, using known time-level values of $\theta^{\prime}$ and $\Pi^{\prime}$. These known values might be either time-level $n$ values or a second-order temporal extrapolation from time levels $n$ and $n-1$.

If the actual atmospheric perturbations from the assumed reference profiles are small then this approach will be both accurate and stable since the terms that are lagged in time will be genuinely small. However, consider what happens when the actual atmospheric vertical profile is observed to have profiles $\theta=\theta_{S}$ and $\Pi=\Pi_{S}$ (which both vary in time) so that the genuinely small, perturbation quantities are $\theta^{\prime \prime} \equiv \theta-\theta_{S}$ and $\Pi^{\prime \prime} \equiv \Pi-\Pi_{S}$. Then the non-linear term becomes

$$
c_{p} \theta^{\prime} \delta_{z} \Pi^{\prime}=c_{p}\left(\theta_{S}-\theta_{r e f}+\theta^{\prime \prime}\right) \delta_{z}\left(\Pi_{S}-\Pi_{r e f}+\Pi^{\prime \prime}\right) .
$$

The terms $\theta_{S}-\theta_{\text {ref }}$ and $\Pi_{S}-\Pi_{r e f}$ are not small, perturbation quantities. Yet in the approach outlined above they are handled in an explicit manner. Noting that the actual atmospheric profiles are not known a priori - they are part of the solution - then unless care is taken this approach can lead to an instability.

The presence of such an instability was first analyzed in the context of a three-time-level HPEs model by [27] and later extended to two-level schemes by [28]. The latter analysis showed that the instability could be avoided by choosing the reference temperature profile to be warmer than any expected atmospheric profile. This is because of the effect that the choice of reference profile has on the propagation speed of gravity waves (the warmer the profile the slower the propagation speed).

Whilst the HPEs only admit Rossby waves and gravity waves, a nonhydrostatic model also admits acoustic waves. It was pointed out in [11] that stability of the acoustic waves requires the reference profile to be colder than any expected atmospheric profile. Essentially because of the different dispersion relation, the choice of a colder reference state slows the speed of acoustic waves [29]. The authors of [11] conclude that stability is therefore difficult to obtain by this method in models that admit both gravity and acoustic waves.

\subsection{Evolving the ENDGame approach}

As a non-hydrostatic compressible model admitting both acoustic and gravity waves, the New Dynamics appears to sidestep the issue raised in section 2.2 by not (explicitly) using a reference profile at all. Instead, by 


\section{T. Benacchio, N. Wood}

using a rather complex set of predictors and correctors (described in [16]), expression (5) is discretized using solely a mix of time level $n$ and $n+1$ quantities. However, the disadvantage of this approach is that there is little control over the complexity of the resulting Helmholtz problem. That used in the New Dynamics has a 45 point stencil which can result in scalability problems on massively parallel computer architectures.

In addition, the choice of the reference profile determines the magnitude of the quantities estimated at the different time levels. As a result, the model prediction depends on the choice of reference profile, the dependence only vanishing asymptotically, as the time step tends to zero. This aspect was investigated in a shallow-water context by [30], the results of which strongly influenced the design of ENDGame.

The approach followed in ENDGame therefore attempts to have good flexibility over which terms are retained in the Helmholtz problem, allowing for a design with improved scalability, whilst reducing the dependence of the scheme on any choice of reference profile. This is achieved in three steps.

- The first step involves the use of the model configuration at the previous time level $t^{n}$ as reference profile. The profile therefore varies time step by time step (though its time dependence is not accounted for within a model time step). This reference profile is used to create terms that are linear in the prognostic quantities.

- The second step involves choosing the linear terms that need to be evaluated implicitly in order to stabilize the model, and hence choosing the form of the Helmholtz problem. The resulting linear terms are simply added and subtracted from the non-linear term in question (see the worked example below).

- The third step involves the iteration of the whole scheme, so that both the non-linear term (that is not part of the Helmholtz problem) and, for specificity, the linear term that was subtracted from it, are evaluated at the previous iteration (not at the previous time step).

As an example, consider again the pressure gradient term (5). Inspired by the linearization used in (6), expression (5) can be rewritten exactly as

$$
\begin{aligned}
G_{A}^{n+1}= & +\left[c_{p} \theta_{r e f} \delta_{z}\left(\Pi_{A}^{n+1}-\Pi_{r e f}\right)+c_{p}\left(\theta_{A}^{n+1}-\theta_{r e f}\right) \delta_{z} \Pi_{r e f}\right] \\
& +c_{p} \theta_{A}^{n+1} \delta_{z} \Pi_{A}^{n+1} \\
& -\left[c_{p} \theta_{r e f} \delta_{z}\left(\Pi_{A}^{n+1}-\Pi_{r e f}\right)+c_{p}\left(\theta_{A}^{n+1}-\theta_{r e f}\right) \delta_{z} \Pi_{r e f}\right]
\end{aligned}
$$

Within an iterative procedure in which the next, unknown iterative value 
is denoted by superscript $(k+1)$, expression (8) is then approximated as:

$$
\begin{aligned}
G_{A}^{n+1} \approx G_{A}^{(k+1)} \equiv & +\left[c_{p} \theta_{r e f} \delta_{z}\left(\Pi_{A}^{(k+1)}-\Pi_{r e f}\right)+c_{p}\left(\theta_{A}^{(k+1)}-\theta_{r e f}\right) \delta_{z} \Pi_{r e f}\right] \\
& +c_{p} \theta_{A}^{(k)} \delta_{z} \Pi_{A}^{(k)} \\
& -\left[c_{p} \theta_{r e f} \delta_{z}\left(\Pi_{A}^{(k)}-\Pi_{r e f}\right)+c_{p}\left(\theta_{A}^{(k)}-\theta_{r e f}\right) \delta_{z} \Pi_{r e f}\right] .
\end{aligned}
$$

The terms involving values at iteration $(k+1)$ are used in forming the Helmholtz operator, the remaining terms, involving values at iteration $(k)$, appear on the right-hand side of the Helmholtz problem.

At convergence (so that for example $\theta^{(k+1)}=\theta^{(k)}$ ) this scheme solves the full non-linear problem, independently of the size of the time step (provided that it is chosen so that the scheme converges). Since $\theta^{n}$ and $\Pi^{n}$ are model solutions and evolve realistically in time, using them as reference profiles for the solution at the next time step means that the model solution is not dependent on some arbitrary initial choice of reference profile. This is true even if the scheme is not iterated to convergence. Additionally, the choice $\theta_{\text {ref }}=\theta^{n}, \Pi_{\text {ref }}=\Pi^{n}$ aids convergence of the scheme.

\subsection{The semi-Lagrangian departure point}

So far it has been assumed that the departure point $\boldsymbol{x}_{D}$ is known. However, except for very simple flows this is not the case and the departure point has to be determined by solving the trajectory or kinematic equation [31]:

$$
\frac{D \boldsymbol{x}}{D t}=\boldsymbol{u} .
$$

In view of the similarity between this equation and equation (1), an obvious method to solve (10) is the discretization given by (4) which, when rearranged, results in:

$$
\boldsymbol{x}_{D}^{n}=\boldsymbol{x}_{A}^{n+1}-\frac{\Delta t}{2}\left(\boldsymbol{u}_{A}^{n+1}+\boldsymbol{u}_{D}^{n}\right) .
$$

An interesting aspect of this equation is that it is doubly implicit: both $\boldsymbol{u}_{D}^{n}$ and $\boldsymbol{u}_{A}^{n+1}$ are unknown until $\boldsymbol{x}_{D}^{n}$ is known. This aspect has led to various approximations being used, for a discussion of some of which see [32]. As analyzed by [33] though, many of these approximations lead to instabilities. The advantage of employing an iterative scheme, as discussed in section 2.3 , is that these issues can be avoided by embedding the departure point calculation within that iterative scheme so that, at convergence, expression (11) is recovered. 


\subsection{The iterative approach employed in ENDGame}

Using the notation of section 2.1, the ENDGame scheme can be summarized for one time step as:

1. Given $F^{n}$ and $\boldsymbol{x}_{D}^{n-1}$, initialize the estimate of the model state vector $F$ at the next time level, denoted by $F^{(0)}$, and also an estimate of $\boldsymbol{x}_{D}^{n}$, denoted by $\boldsymbol{x}_{D}^{n(0)}$;

2. Evaluate the vector $G^{n}$;

3. Outer loop:

(a) Evaluate the next estimate of the departure points, $\boldsymbol{x}_{D}^{n(k+1)}$, from $\boldsymbol{x}_{D}^{n(k)}=\boldsymbol{x}_{A}^{n+1}-\frac{\Delta t}{2}\left(\boldsymbol{u}_{A}^{(k)}+\boldsymbol{u}_{D}^{n(k)}\right)$;

(b) Interpolate $G^{n}$ to those departure points to obtain $G_{D}^{n}$;

(c) Inner loop:

i. Evaluate the latest estimates for $G^{n+1}$, i.e. evaluate $G^{(k)}$ as a function of $F^{(k)}$;

ii. Evaluate the linearized terms required for the Helmholtz problem;

iii. Form and solve the Helmholtz problem for the latest pressure field $\Pi^{(k+1)}$;

iv. Backsubstitute to obtain updated estimates $F^{(k+1)}$;

(d) Repeat inner loop as required;

4. Repeat outer loop as required.

5. Set $F^{n+1}=F^{(k+1)} ; \boldsymbol{x}_{D}^{n}=\boldsymbol{x}_{D}^{n(k+1)}$.

The Helmholtz problem obtained through the nonlinear procedure $H\left(\Pi^{(k+1)}\right)=R$ is solved with a BiCGstab method iterated until $\varepsilon$ reduces below a specified tolerance, where:

$$
\varepsilon=\frac{R M S\left\{D I A G(H)^{-1}\left[H\left(\Pi^{(k+1)}\right)-R\right]\right\}}{R M S\left[D I A G(H)^{-1} H\left(\Pi^{(k+1)}\right)\right]},
$$

where RMS denotes the root-mean-squared value and DIAG denotes the diagonal part of the Helmholtz operator $H$. In the operational setting, the tolerance is set to $\varepsilon=10^{-4}$. Further details are given in [23].

\section{An ENDGame vertical column model}

In this section we describe the results of ENDGame simulations in a simplified setting. As a full analysis of the operational model performance lies outside the scope of this work, we consider a one-dimensional vertical column implementation of the model, and focus on the test case of acoustic 
normal modes of a compressible atmosphere. We refer the reader to the cited work [23] for a complete description of the grid arrangement and features of the numerical scheme.

\subsection{Governing equations}

The momentum, thermodynamic, continuity and trajectory equations in a one-dimensional vertical column of dry air read:

$$
\begin{aligned}
\frac{D w}{D t} & =-c_{p} \theta \frac{\partial \Pi}{\partial z}-g, \\
\frac{D \theta}{D t} & =0, \\
\frac{D \rho}{D t}+\rho \frac{\partial w}{\partial z} & =0, \\
\frac{D z}{D t} & =w,
\end{aligned}
$$

to be solved in the spatio-temporal domain $\left[0, z_{H}\right] \times[0, T] \ni(z, t)$. In (13)-(16), $w$ and $\rho$ denote fluid velocity and dry density, respectively, with the material derivative defined here by $\frac{D}{D t}(\cdot)=\frac{\partial}{\partial t}(\cdot)+w \frac{\partial}{\partial z}(\cdot)$, and $g$ the acceleration due to gravity.

The system is closed by the equation of state for a perfect gas:

$$
\rho=\left(\frac{p_{0}}{R}\right) \frac{\Pi^{(1-\kappa) / \kappa}}{\theta} .
$$

\subsection{Linear analytical solutions}

Equations (13)-(16) are linearized around a motionless isothermal profile in hydrostatic balance with potential temperature:

$$
\theta_{S}=T_{S} \exp \left(\frac{g}{c_{p} T_{S}} z\right)
$$

where $T \equiv T_{S}$, and $\Pi_{S}$ is obtained from the hydrostatic balance equation $\partial \Pi_{S} / \partial z=-g /\left(c_{p} \theta_{S}\right)$. Analytical solutions for the resulting linear perturbations can then be found. (We refer to [34] for a full derivation.) 


\section{T. Benacchio, N. Wood}

The solutions for the linear perturbations are [34]:

(19)

$$
\begin{aligned}
w(z, t) & =-\alpha \imath \omega \frac{(1-\kappa)}{\kappa} \exp (\imath \omega t) \sin \left(\frac{m \pi}{z_{H}} z\right) \exp \left(\frac{z}{2 H}\right), \\
\theta(z, t) & =\alpha \frac{T_{S}(1-\kappa)}{H} \exp (\imath \omega t) \sin \left(\frac{m \pi}{z_{H}} z\right) \exp \left(\frac{1+2 \kappa}{2 H} z\right), \\
\Pi(z, t) & =\alpha \exp (\imath \omega t)\left[\frac{m \pi}{z_{H}} \cos \left(\frac{m \pi}{z_{H}} z\right)-\frac{1-2 \kappa}{2 H} \sin \left(\frac{m \pi}{z_{H}} z\right)\right] \exp \left(\frac{1-2 \kappa}{2 H} z\right), \\
\rho(z, t) & =\alpha \frac{(1-\kappa) p_{0}}{\kappa^{2} c_{p} T_{S}} \exp (\imath \omega t)\left[\frac{m \pi}{z_{H}} \cos \left(\frac{m \pi}{z_{H}} z\right)-\frac{1}{2 H} \sin \left(\frac{m \pi}{z_{H}} z\right)\right] \exp \left(-\frac{z}{2 H}\right),
\end{aligned}
$$

where $z_{H}$ is the top of the domain, $m$ is the internal mode index, and

$$
\omega= \pm c_{s} \sqrt{\left(\frac{m \pi}{z_{H}}\right)^{2}+\frac{1}{4 H^{2}}}, \quad c_{s}^{2}=\frac{R T_{S}}{1-\kappa}, \quad H=\frac{R T_{S}}{g}
$$

denote the temporal frequency, the speed of sound, and the scale height, respectively. $p_{0}=1.0 \mathrm{E} 5 \mathrm{~Pa}$ denotes the pressure at sea level, and $\kappa=R / c_{p}$. To obtain the results shown below, unless otherwise stated the constant of proportionality $\alpha$, which determines the amplitude of the perturbation, is set to unity, in agreement with [34].

Because of their easy setup, normal modes allow the implementation and investigation of a number of configurations and choices, and the evaluation of the respective accuracy and stability properties, prior to introducing them in fully-fledged NWP models [35]. In addition, normal mode analyses have been employed in assessing the impact of approximations to the fully compressible, deep atmosphere equations in [36-39].

Here we follow in the steps of the analysis in $[33,34]$ and report numerical results of ENDGame runs, evaluating the impact of different modelling choices. A standard direct method (Thomas's algorithm) was used for the solution of the tridiagonal solve in the simplified one-dimensional setting.

\subsection{Numerical experiments}

Simulations of the ENDGame vertical column model are initialized using the expressions (19) evaluated at $t=0$ and sampled spatially where required.

We consider a basic configuration with model top $z_{H}=40000 \mathrm{~m}$, final time $T=100 \mathrm{~s}$, grid spacing $\Delta z=200 \mathrm{~m}$, time step $\Delta t=1 \mathrm{~s}$, two outer and two inner loop iterations, iterative procedure for the trajectory calculation, isothermal physical background state with $T_{S}=290 \mathrm{~K}$, semi-implicit 
reference state chosen as the atmospheric state at the previous time level, as discussed in section 2.3, and for simplicity we use a uniformly spaced grid. Additionally, cubic Lagrange interpolation is used for evaluation of quantities at the departure points.

Numerical results for two different wave indices confirm the accuracy of the model as compared with the linear analytical solutions (Figures 1 and 2 ). For reference, we have $\omega \Delta t \approx 0.109$ for index $m=4, \omega \Delta t \approx 0.242$ for index $m=9$.
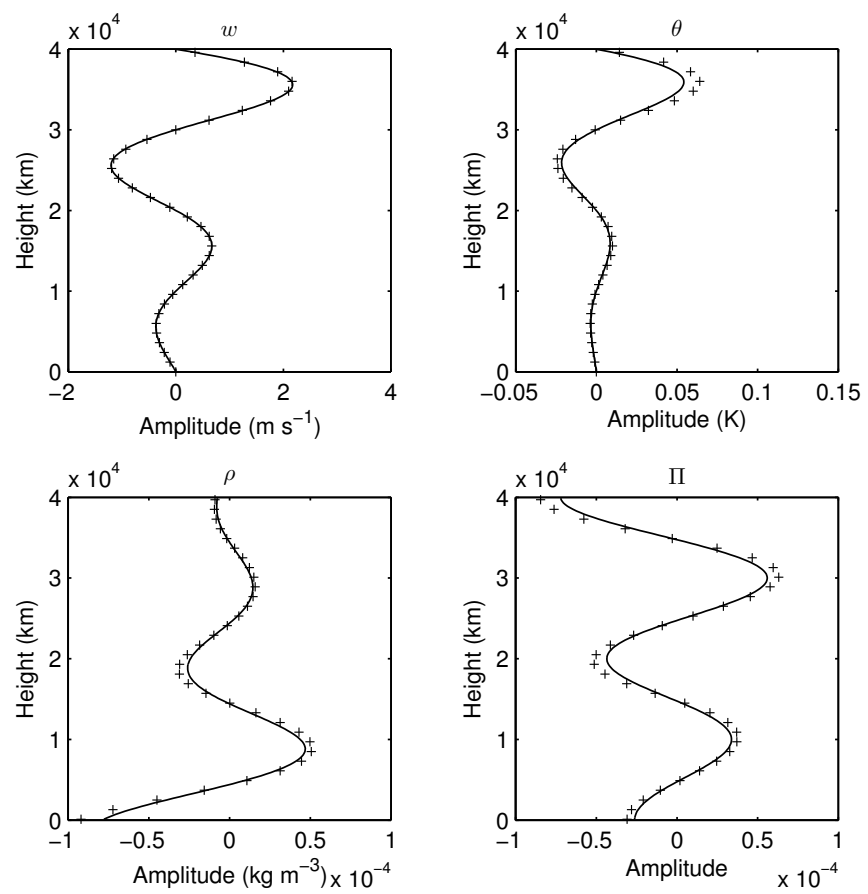

Figure 1. Perturbations of $w, \theta, \rho$, and $\Pi$ over a $40 \mathrm{~km}$-deep isothermal atmosphere at final time $T=100 \mathrm{~s}$ computed by the ENDGame vertical column model. The solution is obtained evolving the initial data of the internal normal mode with index $m=4$ with uniform grid spacing $\Delta z=200 \mathrm{~m}$, time step $\Delta t=1 \mathrm{~s}$, two outer and two inner loop iterations, iterative procedure for the trajectory calculation, and $T_{S}=290 \mathrm{~K}$. Numerical solution (pluses) and linear analytical solution (solid line). For the numerical solution, one in seven markers is plotted for readability.

Moreover, the discretization error with respect to the linear analytical solution decreases with decreasing time step (Figure 3). Numerical experiments display temporal and spatial second-order accuracy, with the spatial error asymptotically dominating in the temporal convergence studies (Figures 4,5 , and 6 ).

Experiments were also carried out with a time step ten times larger than 


\section{T. Benacchio, N. Wood}
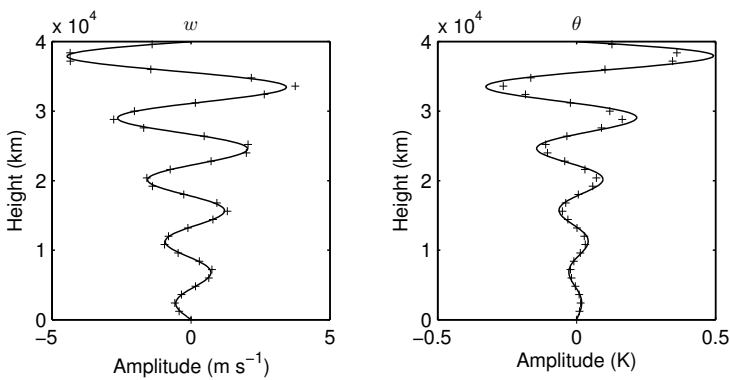

Figure 2. As in Figure 1, but with index $m=9$.
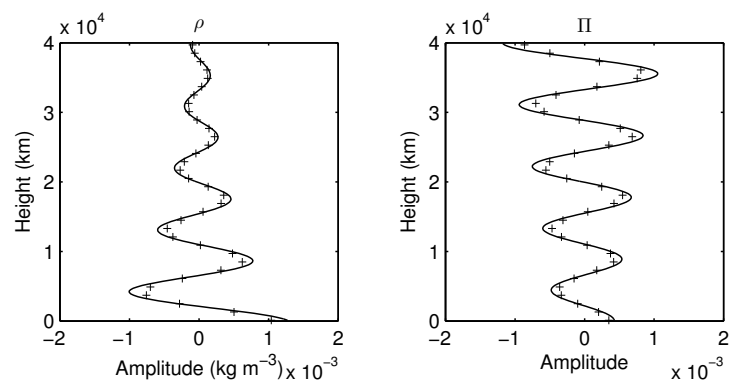

the standard one (not shown). A slowing of the phase was observed because of the poor temporal resolution of the wave frequency. However, with the operational 2-by-2 outer-inner loop configuration an unexpected damping effect was found. This was discovered to be due to the updating of some terms in the outer loop which is done to enhance the stability (see [23] for details). Increasing the outer loop count to 4 was sufficient to recover an undamped result but still with the expected slowing of the phase.

A note of caution is in order regarding the comparison of these results with the results in [33]. The model in [33] solves the linearized discrete equations, whereas the results here refer to the direct solution of the nonlinear model (13). As such, an error is inherent by taking expressions (19) as analytical solutions for the non-linear model. However, as is apparent in Figure 1, we have $\theta^{\prime} / \theta_{S}, \Pi^{\prime} / \Pi_{S} \sim \mathcal{O}\left(10^{-3}\right)$, whilst $w / c_{s} \sim \mathcal{O}\left(10^{-2}\right)$. It is therefore expected that errors due to the nonlinear terms will be at least two orders of magnitude smaller than the linear terms. This effect is therefore negligible in terms of the convergence plots of Figures 4-6.

\subsection{Impact of the trajectory calculation strategy}

As seen in Section 2.4 above, ENDGame features an iterative procedure for the trajectory calculation. In the context of the vertical column model, the trajectory equation is

$$
z_{D}^{n(K)}=z^{n+1}-\frac{\Delta t}{2}\left\{w^{n+1}+w^{n}\left[z_{D}^{n(K-1)}\right]\right\} \quad K=1,2, \ldots
$$



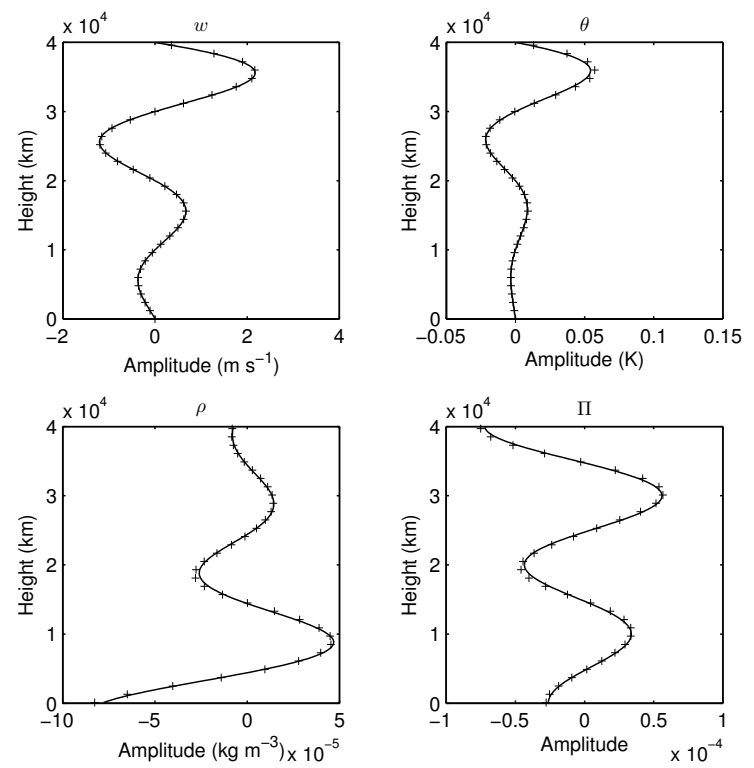

Figure 3. As in Figure 1, but with time step $\Delta t=0.25 \mathrm{~s}$.

The iteration (21) is initialized with the value at the previous time step, $z_{D}^{n(0)}=z_{D}^{n-1}$, and solved separately from the outer-inner loop iteration for the full non-linear problem described in section 2.5. Therefore, the iteration count $K$ in (21) is distinct from the $k$ index used in section 2. In contrast to the cubic interpolation used elsewhere in model, the value of $w^{n}$ at the departure point in (21) is found by linear interpolation.

The ENDGame strategy is compared with a two-term extrapolation strategy for the velocity as in the operational setting of the New Dynamics dynamical core $[16,33]$, whereby the curly bracket in $(21)$ is replaced by $w_{M}^{n+1 / 2}$ computed as:

$$
w_{M}^{n+1 / 2} \equiv \frac{3}{2} w_{M}^{n}-\frac{1}{2} w_{M}^{n-1},
$$

where the subscript $M$ denotes the midpoint of the trajectory from $z_{D}$ to $z_{A}$ to which the value of the velocity is linearly interpolated in space.

The solution obtained using extrapolation is more diffusive and less accurate than the one obtained with the interpolation strategy employed in ENDGame (Figure 7).

\subsection{Impact of the loop number in the iterating procedure}

The semi-implicit model used in ENDGame features a pressure problem constructed iteratively at each time step with a double loop structure. If iterated to convergence, the target discretization (4) is obtained. However, 

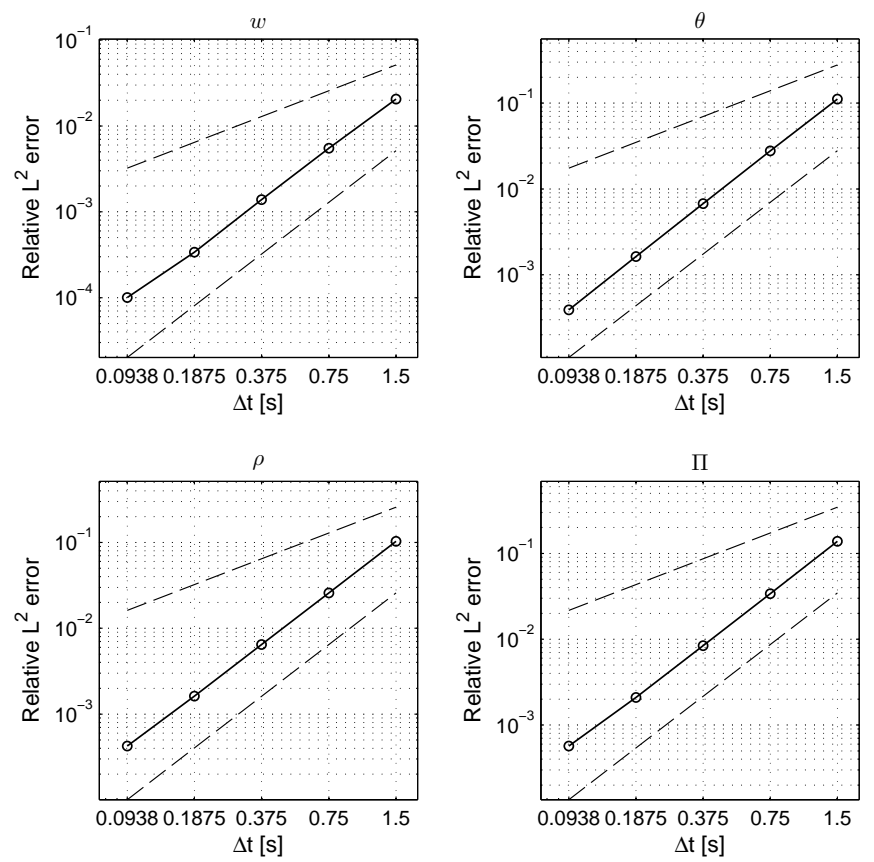

Figure 4. Convergence study for the ENDGame vertical column model in the basic setting, see Figure 1. Relative $L^{2}$ errors of the computed $w, \theta, \rho$, and $\Pi$ at time $T=75 \mathrm{~s}$ with respect to the linear analytical solution are displayed. In the initial data (19), the constant $\alpha$ has been divided by a factor of 100 to mitigate the impact of non-linear effects, and a fixed reference state is used. For this simulation, the acoustic Courant number is held fixed at 0.64 , which corresponds to a spatial resolution $\Delta z=800 \mathrm{~m}$ for $\Delta t=1.5 \mathrm{~s}$. The dashed lines denote first and second order convergence.

the operational configuration of two outer and two inner loops constitutes a trade-off between accuracy and constraints on execution time. Figures 8 and 9 show differences between the operational configuration and one using eight outer and eight inner loops. For the current test case, it is seen that there is very little sensitivity to increasing the number of outer and inner loops. This suggests that in this setup the model is close to convergence with only two outer and two inner loops.

\section{Conclusion}

In this paper, an overview of the semi-implicit semi-Lagrangian method in an NWP context has been given, drawing on the legacy of the dynamical cores in use at the Met Office. Different aspects and modelling choices in the framework of the currently operational ENDGame dynamical core have been considered. Numerical results in a simplified vertical column model 
SISL modelling: a Met Office perspective
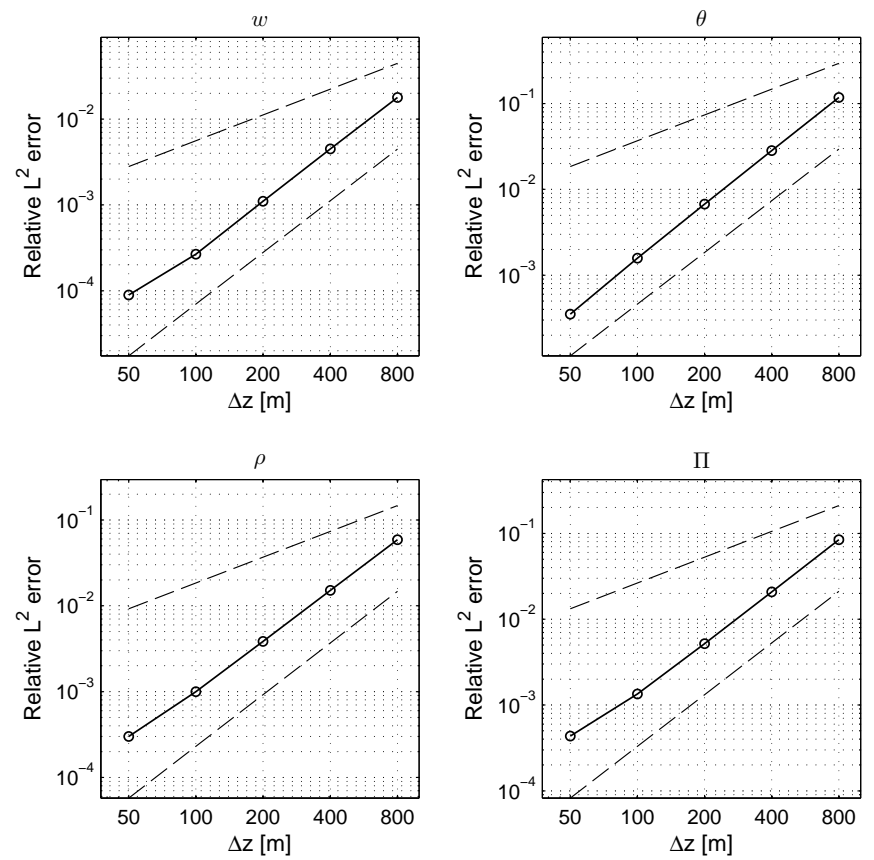

Figure 5. As in Figure 4 , but for fixed time step $\Delta t=0.046875 \mathrm{~s}$ and varying grid size $\Delta z$.
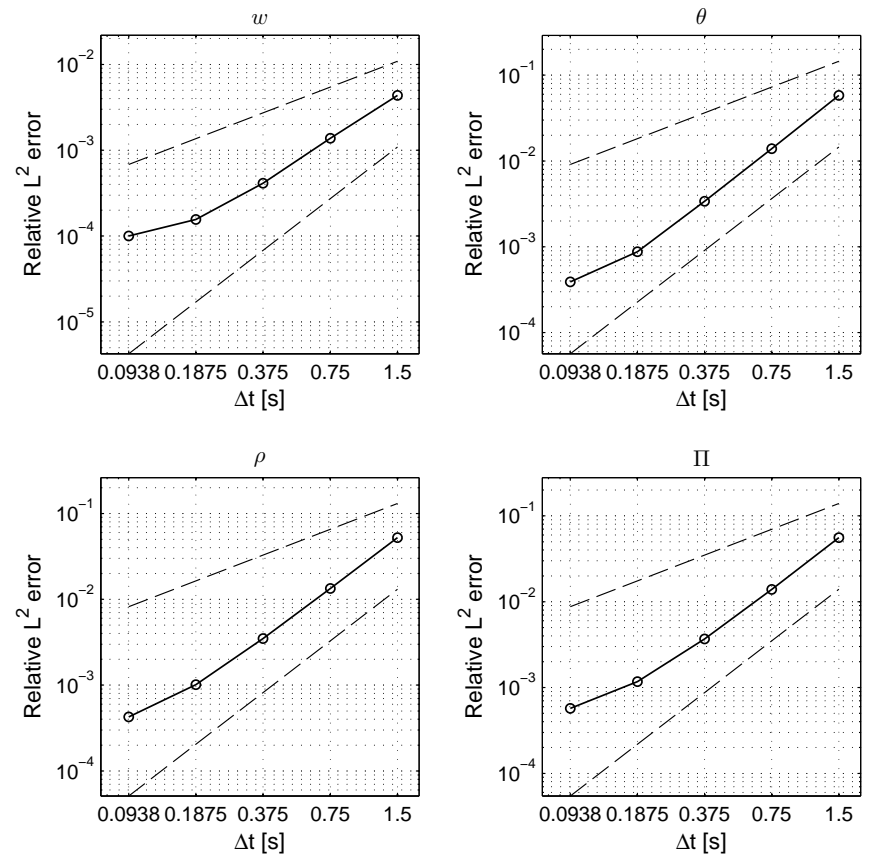

Figure 6. As in Figure 4 , but for fixed grid size $\Delta z=50 \mathrm{~m}$ and varying time step $\Delta t$.

have demonstrated the model performance on test cases with normal mode initial data. 

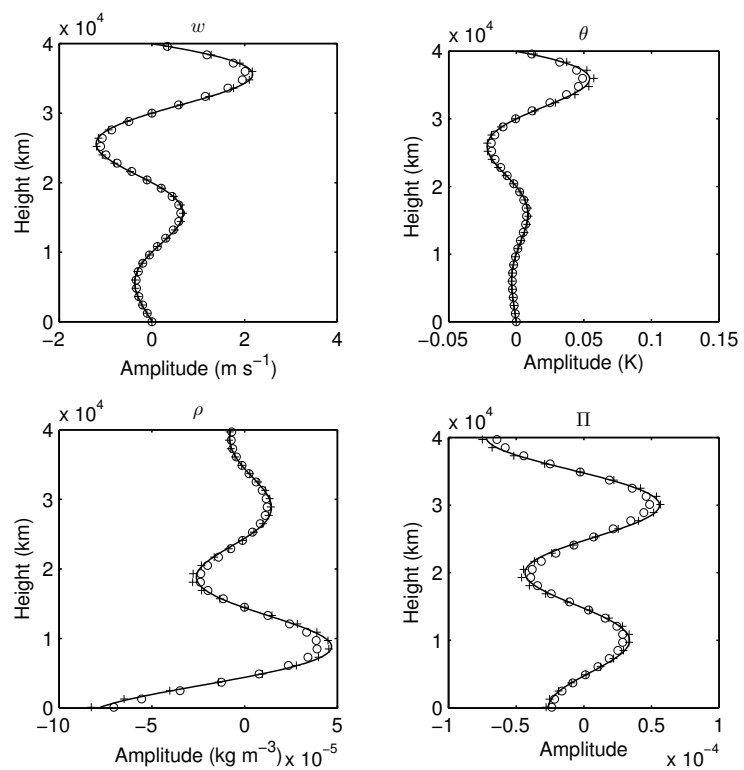

Figure 7. As in Figure 3, using different methods for the trajectory calculation: iterative interpolation (pluses), two-term extrapolation (circles), linear analytical solution (solid line).

For the sake of conciseness this paper has not reported in detail all the modelling choices in ENDGame that have enabled enhanced performance over the New Dynamics dynamical core. Further details can be found in [23], but two examples are: the Lagrangian treatment of the continuity equation and the choice of how to stagger variables with respect to the Poles in the latitude-longitude grid. These features, together with the discussed iterative procedure for handling the non-linearity and the interpolation strategy for the trajectory computation, have allowed for a discretization with reduced temporal damping and reduced polar filtering. As a result, the model is more robust, stable and scalable (see [40] for further details). 
SISL modelling: a Met Office perspective
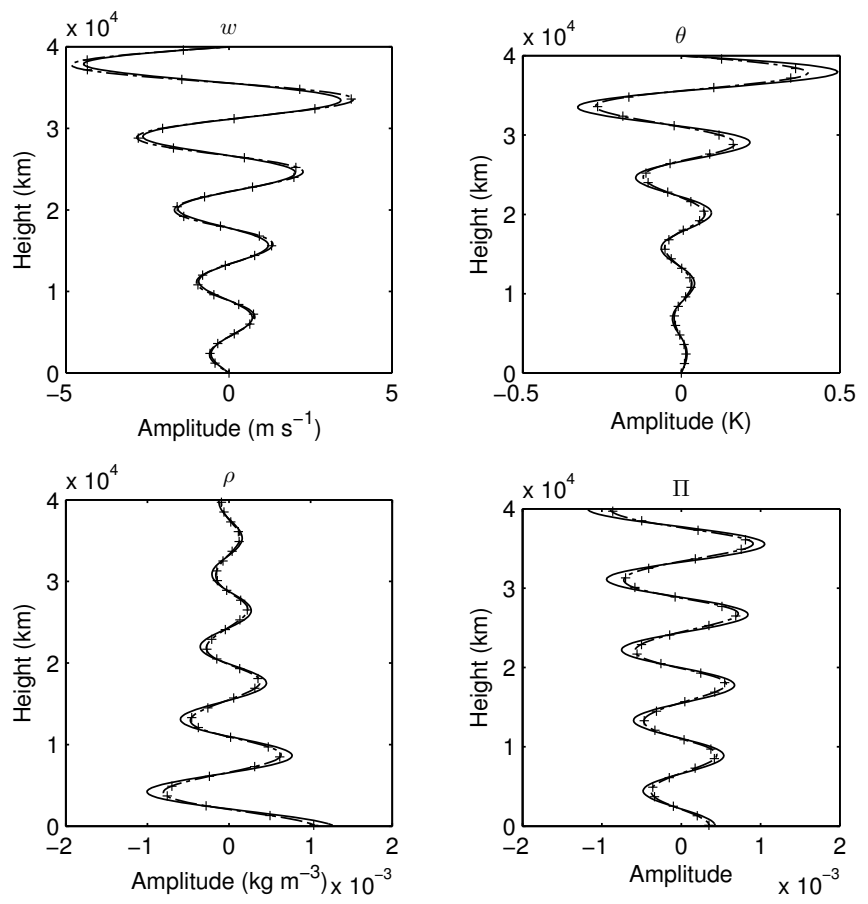

Figure 8. As in Figure 2, using different number of outer and inner loop: 2-by-2 (pluses), 8-by-8 (dashed-dotted line), linear analytical solution (solid line).
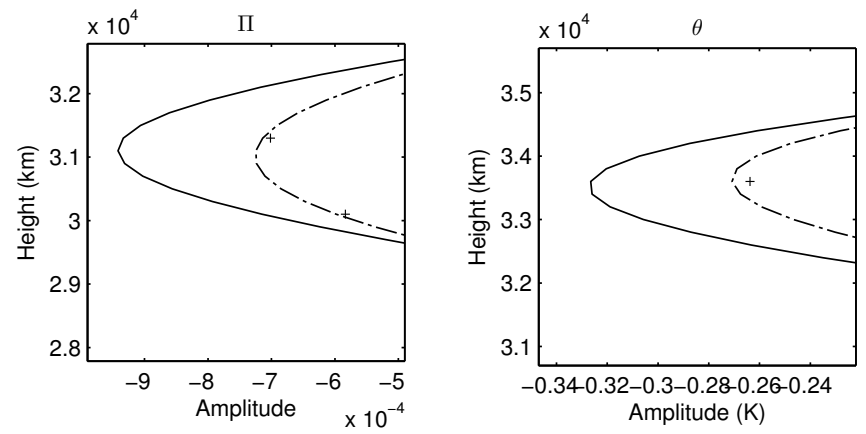

Figure 9. Close-up of the uppermost crests of $\Pi$ and $\theta$ modes in Figure 8.

\section{REFERENCES}

1. C. Temperton, M. Hortal, and A. J. Simmons, A two-time-level semiLagrangian global spectral model, Quarterly Journal of the Royal Meteorological Society, vol. 127, pp. 111-126, 2001.

2. P. Courtier, C. Freydier, J. F. Geleyn, F. Rabier, and M. Rochas, The ARPEGE project at Météo-France, in ECMWF Workshop on Numerical Methods in Atmospheric Modelling, Vol. II, 2, pp. 193-231, 1991. 


\section{T. Benacchio, N. Wood}

3. J. Côté, S. Gravel, A. Méthot, A. Patoine, M. Roch, and A. Staniforth, The operational CMC-MRB global environmental multiscale (GEM) model. Part I: Design considerations and formulation, Monthly Weather Review, vol. 126, pp. 1373-1395, 1998.

4. D. Chen, J. Xue, X. Yang, H. Zhang, X. Shen, J. Hu, Y. Wang, L. Ji, and J. Chen, New generation of multi-scale NWP system (GRAPES): general scientific design, Chinese Science Bulletin, vol. 53, pp. 3433$3445,2008$.

5. Y. Takeuchi and Others, Outline of the operational numerical weather predic-

tion at the Japan Meteorological Agency. Appendix to WMO Technical Progress Report on the Global Data-processing and Forecasting System (GDPFS) and Numerical Weather Prediction (NWP) Research, tech. rep., JMA, 2013. Available at: http://www.jma.go.jp/jma/jma-eng/ jma-center/nwp/outline2013-nwp/pdf/outline2013_all.pdf (Last access: 8 May 2015).

6. M. Tolstykh, Variable resolution global semi-Lagrangian atmospheric model, Russian Journal of Numerical Analysis and Mathematical Modelling, vol. 18, pp. 347-361, 2003.

7. M. Zhao, I. Held, S. Lin, and G. Vecchi, Simulations of global hurricane climatology, interannual variability, and response to global warming using a 50-km resolution gcm., Journal of Climate, vol. 22, pp. 6653-6678, 2009.

8. R. B. Neale and Others, Description of the NCAR Community Atmosphere Model (CAM5.0), tech. rep., NCAR, 2012. Available at: http://www.cesm.ucar.edu/models/cesm1.0/cam/docs/ description/cam5_desc.pdf (Last access: 17 August 2015).

9. A. Brown, S. Milton, M. Cullen, B. Golding, J. Mitchell, and A. Shelly, Unified modeling and prediction of weather and climate: A 25-year journey, Bulletin of the American Meteorological Society, vol. 93, pp. 18651877, 2012.

10. A. Staniforth and J. Côté, Semi-Lagrangian integration schemes for atmospheric models - a review, Monthly Weather Review, vol. 119, pp. 2206-2223, 1991.

11. P. Bénard, Stability of semi-implicit and iterative centered-implicit time discretizations for various equation systems used in NWP, Monthly Weather Review, vol. 131, pp. 2479-2491, 2003.

12. A. Staniforth and J. Thuburn, Horizontal grids for global weather pre- 
diction and climate models: a review, Quarterly Journal of the Royal Meteorological Society, vol. 138, pp. 1-26, 2012.

13. M. J. P. Cullen, Modelling atmospheric flows, Acta Numerica, vol. 16, pp. 67-154, 2007.

14. M. J. P. Cullen, T. Davies, M. H. Mawson, J. A. James, S. C. Coulter, and A. Malcolm, An overview of numerical methods for the next generation UK NWP and climate model, in Numerical Methods in Atmospheric Modelling, The André Robert memorial volume (C. Lin, R. Laprise, and H. Ritchie, eds.), (Ottawa, Canada), pp. 425-444, Canadian Meteorological and Oceanographical Society, 1997.

15. M. C. Tapp and P. W. White, A non-hydrostatic mesoscale model, Quarterly Journal of the Royal Meteorological Society, vol. 102, pp. 277296, 1992.

16. T. Davies, M. J. P. Cullen, A. J. Malcolm, M. H. Mawson, A. Staniforth, A. White, and N. Wood, A new dynamical core for the Met Office's global and regional modelling of the atmosphere, Quarterly Journal of the Royal Meteorological Society, vol. 131, pp. 1759-1782, 2005.

17. A. A. White and R. A. Bromley, Dynamically consistent, quasihydrostatic equations for global models with a complete representation of the Coriolis force, Quarterly Journal of the Royal Meteorological Society, vol. 121, pp. 399-418, 1995.

18. A. A. White, B. J. Hoskins, I. Roulstone, and A. Staniforth, Consistent approximate models of the global atmosphere: shallow, deep, hydrostatic, quasi-hydrostatic and non-hydrostatic, Quarterly Journal of the Royal Meteorological Society, vol. 131, pp. 2081-2107, 2005.

19. R. Laprise, The Euler equations of motion with hydrostatic pressure as an independent variable, Monthly Weather Review, vol. 120, pp. 197207, 1992.

20. N. Wood and A. Staniforth, The deep-atmosphere Euler equations with a mass-based vertical coordinate, Quarterly Journal of the Royal Meteorological Society, vol. 129, pp. 1289-1300, 2003.

21. P. R. Bannon, C. H. Bishop, and J. B. Kerr, Does the surface pressure equal the weight per unit area of a hydrostatic atmosphere?, Bulletin of the American Meteorological Society, vol. 78, pp. 2637-2642, 1997.

22. M. Zerroukat, N. Wood, and A. Staniforth, SLICE-S: A SemiLagrangian Inherently Conserving and Efficient scheme for transport problems on the sphere, Quarterly Journal of the Royal Meteorological Society, vol. 130, pp. 2649-2664, 2004. 


\section{T. Benacchio, N. Wood}

23. N. Wood, A. Staniforth, A. White, T. Allen, M. Diamantakis, M. Gross, T. Melvin, C. Smith, S. Vosper, M. Zerroukat, and J. Thuburn, An inherently mass-conserving semi-implicit semi-Lagrangian discretization of the deep-atmosphere global non-hydrostatic equations, Quarterly Journal of the Royal Meteorological Society, vol. 140, pp. 1505-1520, 2014.

24. K.-S. Yeh, J. Côté, S. Gravel, A. Méthot, A. Patoine, M. Roch, and A. Staniforth, The CMC-MRB Global Environmental Multiscale (GEM) model. Part III: Nonhydrostatic formulation, Monthly Weather Review, vol. 130, pp. 339-356, 2002.

25. M. J. P. Cullen, Alternative implementations of the semi-Lagrangian semi-implicit schemes in the ECMWF model, Quarterly Journal of the Royal Meteorological Society, vol. 127, pp. 2787-2802, 2001.

26. M. Diamantakis, T. Davies, and N. Wood, An iterative timestepping scheme for the Met Office's semi-implicit semi-Lagrangian nonhydrostatic model, Quarterly Journal of the Royal Meteorological Society, vol. 133, pp. 997-1011, 2007.

27. A. J. Simmons, B. J. Hoskins, and D. M. Burridge, Stability of the semiimplicit method of time integration, Monthly Weather Review, vol. 106, pp. 405-412, 1978.

28. A. J. Simmons and C. Temperton, Stability of a two-time-level semiimplicit integration scheme for gravity wave motion, Monthly Weather Review, vol. 125, pp. 600-615, 1997.

29. J. Thuburn, Vertical discretizations giving optimal representation of normal modes: Sensitivity to the form of the pressure-gradient term, Quarterly Journal of the Royal Meteorological Society, vol. 132, pp. 2809-2825, 2006.

30. J. Thuburn, M. Zerroukat, N. Wood, and A. Staniforth, Coupling a mass-conserving semi-Lagrangian scheme (SLICE) to a semi-implicit discretisation of the shallow-water equations: minimizing the dependence on a reference atmosphere, Quarterly Journal of the Royal Meteorological Society, vol. 136, pp. 146-154, 2010.

31. A. Staniforth, A. White, and N. Wood, Analysis of semi-Lagrangian trajectory computations, Quarterly Journal of the Royal Meteorological Society, vol. 129, pp. 2065-2085, 2003.

32. N. Wood, A. A. White, and A. Staniforth, Treatment of vector equations in deep-atmosphere, semi-Lagrangian models. II: Kinematic equation, Quarterly Journal of the Royal Meteorological Society, vol. 136, pp. 507- 


\section{SISL modelling: a Met Office perspective}

$516,2010$.

33. E. Cordero, N. Wood, and A. Staniforth, Impact of semi-Lagrangian trajectories on the discrete normal modes of a non-hydrostatic verticalcolumn model, Quarterly Journal of the Royal Meteorological Society, vol. 131, pp. 93-108, 2005.

34. E. Cordero, A. Staniforth, and N. Wood, Normal mode analysis of the New Dynamics, tech. rep., FR Technical Report No. 393, 2002. Available at: http://www.metoffice.gov.uk/media/pdf/c/9/ FRTR393-wontconvert.pdf (Last access 13 May 2015).

35. J. Thuburn, N. Wood, and A. Staniforth, Normal modes of deep atmospheres. I: Spherical geometry, Quarterly Journal of the Royal Meteorological Society, vol. 128, pp. 1771-1792, 2002.

36. T. Davies, A. Staniforth, N. Wood, and J. Thuburn, Validity of anelastic and other equation sets as inferred from normal-mode analysis, Quarterly Journal of the Royal Meteorological Society, vol. 129, pp. 27612775,2003 .

37. A. Arakawa and C. S. Konor, Unification of the anelastic and quasihydrostatic systems of equations, Monthly Weather Review, vol. 137, pp. 710-726, 2009.

38. J. K. Dukowicz, Evaluation of various approximations in ocean and atmospheric modeling based on an exact treatment of gravity wave dispersion, Monthly Weather Review, vol. 141, pp. 4487-4506, 2013.

39. T. Dubos and F. Voitus, A semihydrostatic theory of gravity-dominated compressible flow, Journal of the Atmospheric Sciences, vol. 71, pp. 4621-4638, 2014.

40. D. Walters, N. Wood, S. Vosper, and S. Milton, ENDGame: A new dynamical core for seamless atmospheric prediction, tech. rep., Met Office, 2014. Available at: http://www.metoffice.gov.uk/media/pdf/s/h/ ENDGameGOVSci_v2.0.pdf (Last access: 13 May 2015). 